\title{
ON $k$-SPACES
}

\section{D. WEDDINGTON}

One of our first interests in the study of $k$-spaces (spaces with the weak (=finest) topology generated by their compact subsets) was in the determination of those subsets of a topological space which are themselves $k$-spaces. If a subset $A$ of a space $X$ is a $k$-space, then a subset of $A$ is closed in $A$ if it intersects every compact subset $K$ of $X$ in a set closed in $A \cap K$. If a subset $A$ which is not necessarily a $k$-space has this property relative to the compact subsets of $X$, then we say that $A$ has property (k). Throughout this paper, unless exception is noted, we shall assume that compact subsets are closed. A subspace $A$ of a space $X$ is a $k$-space if and only if $A$ has property (k) and $A \cap K$ is a $k$-space for each compact subset $K$ of $X$ (Theorem 1 ). It follows that an open subset of a $k$-space in which compact subsets are regular is itself a $k$-space. The case when the $k$-space is Hausdorff was first established independently in [6] by a different proof. This result is similar to a theorem of N. E. Steenrod [7] (an open subset of a $k$-space is a $k$-space if it is a "regular" open set). The class of subspaces of a space $X$ having property (k) is of interest in itself. For example, every subset of $X$ has property (k) if and only if for any set $A \subset X$ and any point $x \in \bar{A}$, there exists a compact set $K$ such that $x \in \overline{A \cap K}$ (Theorem 2). A. Arhangel'skiǐ [1] calls spaces with this property $k^{\prime}$-spaces. Analogous to this theorem is a result proved by Arhangel'skiǔ [2] which states that a space $X$ is an hereditary $k$-space if and only if $X$ is a Fréchet-Urysohn space.

THEOREM 1. A subset $A$ of a space $X$ is a $k$-space if and only if (1) $A$ has property (k), (2) $A \cap K$ is a $k$-space for each compact subset $K$ of $X$.

Proof. If $A$ is a $k$-subspace of $X$, then $A$ clearly has property (k) and $A$ meets each compact subset of $X$ in a closed subset of $A$, hence in a $k$-subspace of $A$. Conversely, let $U$ be a subset of $A$ which intersects every compact subset of $A$ in a closed set and let $C$ be a compact subset of $X$. We have that $A \cap C$ is a $k$-space; thus $U \cap C$ is closed in $A \cap C$. Since $A$ has property (k), $U$ is then closed in $A$ and $A$ is a $k$-space.

COROLlaRY 1.1. Every open subset of a $k$-space in which compact subsets are regular is a $k$-space. 1969.

Received by the editors November 26, 1968 and, in revised form, February 14, 
Proof. Let $V$ be an open subset of a $k$-space $X$ and let $U$ be a subset of $V$ which intersects every compact subset $K$ of $X$ in an open subset of $V \cap K$. Then $U \cap K$ is open in $K$. Hence $U$ is open in $X$ and $V$ has property (k). Since $V \cap K$ is locally compact it is also a $k$-space. Thus $V$ is a $k$-space.

COROllaRy 1.2. If $X$ is a space in which compact subsets are regular and every point of $X$ is interior to a $k$-subspace of $X$, then $X$ is a $k$-space.

Proof. Let $A$ be a subset of $X$ which intersects every compact subset of $X$ in a closed set. Let $x \in \bar{A}$ and let $U$ be an open $k$-subspace of $X$ containing $x$. Now, let $K$ be a compact subset of $U \cap \bar{A}$. Since $K \cap A$ is closed, $K \cap(A \cap U)$ is closed in $U \cap \bar{A}$. Thus $A \cap U$ is closed in $U \cap \bar{A}$. Therefore, since

$$
x \in \mathcal{L} \cap \bar{A} \cap \overline{A \cap U}=.1 \cap U,
$$

$A$ is closed and $X$ is a $k$-space.

It is easily seen that the separation in Corollary 1.1 cannot be removed. Consider a $T_{1}$ non- $k$-space $Y$. Compactify $Y$ by adjoining an additional point $x$ and defining neighborhoods of $x$ to be complements of finite subsets of $Y . Y \cup\{x\}$ is a compact $T_{1}$ space and $Y$ is an open subset.

In Theorem 1, note that the assumption that compact subsets of $X$ are closed is needed only in the sufficiency to obtain (2). The following example, due to the referee of this paper, shows that (2) is not obtainable without such an assumption.

Let $Y$ be a $T_{1}$ space which is not a $k$-space, $Y^{*}$ is one-point compactification, and let $X=Y \cup\left\{x_{1}, x_{2}\right\}$ be the quotient formed by identifying the copies of $Y$ in the disjoint union of two copies of $Y^{*}$. Then $X, X_{1}=Y \cup\left\{x_{1}\right\}$ and $X_{2}=Y\left\{x_{2}\right\}$ are compact, while $X_{1} \cap X_{2}$ $=Y$ is not a $k$-space.

By the following examples we will show the independence of the two conditions in Theorem 1.

(1) Let $R=$ Reals. Then $R^{R}$ is not a $k$-space [4] but it is a completely regular Hausdorff space. Thus it is embeddable in a compact Hausdorff space. In Theorem 2 we show that every subset of a compact Hausdorff space has property (k).

(2) In [3] we show that, if $X$ is a nondiscrete $T_{1}$ space, then there exists a $k^{\prime}$-space $Y$ such that $X \times Y$ is not a $k^{\prime}$-space. In particular if $X=\{1,2, \cdots, \omega\}$, with the order topology, and $Y=(I \times I) \cup\{z\}$ where $I$ is the positive integers, $I \times I$ discrete, and neighborhoods of $z$ are complements of sets consisting of a finite number of elements in $\{(m, n): n=1,2, \cdots\}$ for each $m$, and $C=U_{i<\omega}\{(i, n, i): n=1$, 
$2, \cdots\}$, then $(\omega, z) \in \bar{C}$ and $(\omega, z) \notin \overline{C \cap K}$ for any compact subset $K$ of $X \times Y$. Let $C^{\prime}=C \cup\{(\omega, z)\}$. It follows without much difficulty that $C^{\prime} \cap K$ is a $k$-space for each compact subset $K$ of $X \times Y$ and that $C^{\prime}$ is not a $k$-space.

It is readily verified that $k^{\prime}$-spaces are $k$-spaces. But there are $k$ spaces which are not $k^{\prime}$-spaces (see [1], [3], [5]). We have, however, the following characterization of a $k^{\prime}$-space.

Theorem 2. A space $X$ is a $k^{\prime}$-space if and only if every subset of $X$ has property (k).

Proof. Let $A$ be a subset of $X$ and $U$ a subset of $A$ which meets every compact subset of $X$ in a set closed in $A$. Since every limit point of $U$ in $A$ is a limit point of $U$ intersected with some compact set, it follows that $U$ is closed in $A$ and $A$ has property (k). Conversely, suppose $X$ is not a $k^{\prime}$-space. Thus there is a subset $A$ of $X$ and a point $x \in \bar{A}-A$ which is not a limit point of the intersection of $A$ with any compact subset of $X$. If $K$ is a compact subset of $X$, then

$$
A \cap K=\overline{A \cap K} \cap[K \cap(A \cup\{x\})] .
$$

Since $A \cup\{x\}$ has property (k), $A$ is closed in $A \cup\{x\}$, which contradicts the fact that $x \notin A$.

Theorem 3. $A$ subspace $A$ of a $k^{\prime}$-space $X$ is a $k^{\prime}$-space if and only if $A \cap K$ is a $k^{\prime}$-space for each compact subset $K$ in $X$.

Proof. Let $K$ be a compact subset of $X$ and let $B$ be a subset of $A \cap K$. Since every limit point of $B$ in $A \cap K$ is clearly a limit point of $B$ in $A$, it follows that $A \cap K$ is a $k^{\prime}$-space. Conversely, if $B$ is a subset of $A$ and $x$ is a limit point of $B$ in $A$, then there is a compact set $K$ such that

$$
x \in \bar{B} \cap K \cap A \subset \bar{B} \cap(A \cap K) .
$$

Thus there is a compact subset $C$ of $A$ for which

$$
x \in \overline{B \cap C} \text {. }
$$

Hence $A$ is a $k^{\prime}$-space.

Corollary 3.1. Let $X$ be a space in which compact subsets are regular. (1) If $X$ is a $k^{\prime}$-space, then every open subset of $X$ is a $k^{\prime}$-space. (2) If each point of $X$ is interior to a $k^{\prime}$-space, then $X$ is a $k^{\prime}$-space.

\section{Bibliography}

1. A. V. Arhangel'skir, Bicompact sets and the topology of spaces, Soviet Math. Dokl. 4 (1963), 561-564. 
2. - A characterization of very k-spaces, Czechoslovak Math. J. 18 (93) (1968), 392-395.

3. R. W. Bagley and D. D. Weddington, Products of $k^{\prime}$-spaces, Proc. Amer. Math. Soc. 22 (1969), 392-394.

4. J. L. Kelley, General topology, Van Nostrand, New York, 1955.

5. N. L. Noble, $k$-spaces and some generalizations, Doctoral Dissertation, University of Rochester, Rochester, N. Y., 1967.

6. R. Pupier and A. Roux, Seminaire de Topologie Generale, Les Espaces de Kelley et de DeGroot, Applications aux espaces localement compacts, Dept. de Mathematiques, Faculte des Sciences de Lyon, p. 16.

7. N. E. Steenrod, A convenient category of topological spaces, Michigan Math. J. 14 (1967), 133-152.

UNIVERSITY OF MiAMI 